\title{
Leçons d'anatomie. Pour une histoire naturelle des images chez Walter Benjamin
}

Muriel Pic

\section{(2) OpenEdition}

12 Journals

Édition électronique

URL : http://journals.openedition.org/imagesrevues/409

DOI : 10.4000/imagesrevues.409

ISSN : 1778-3801

Éditeur :

Centre d'Histoire et Théorie des Arts, Groupe d'Anthropologie Historique de l'Occident Médiéval, Laboratoire d'Anthropologie Sociale, UMR 8210 Anthropologie et Histoire des Mondes Antiques

\section{Référence électronique}

Muriel Pic, «Leçons d'anatomie. Pour une histoire naturelle des images chez Walter Benjamin », Images Re-vues [En ligne], Hors-série 2 | 2010, mis en ligne le 01 janvier 2010, consulté le 03 février 2021. URL : http://journals.openedition.org/imagesrevues/409 ; DOI : https://doi.org/10.4000/ imagesrevues.409

Ce document a été généré automatiquement le 3 février 2021

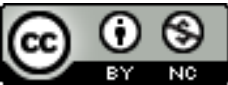

Images Re-vues est mise à disposition selon les termes de la Licence Creative Commons Attribution Pas d'Utilisation Commerciale 4.0 International. 
Leçons d'anatomie. Pour une histoire naturelle des images chez Walter Benjamin

Muriel Pic

J'apprends à voir'1. 
1 L'écrivain W.G. Sebald (1944-2003) situe sa démarche littéraire dans une «histoire naturelle de la destruction $»^{2}$. Cette appréhension de l'histoire comme histoire naturelle ou histoire de la nature, il l'hérite de Walter Benjamin. Elle s'impose, en premier lieu, chez W. G. Sebald comme chez W. Benjamin, grâce à des images empruntées au domaine naturaliste, principalement l'opération anatomique, le

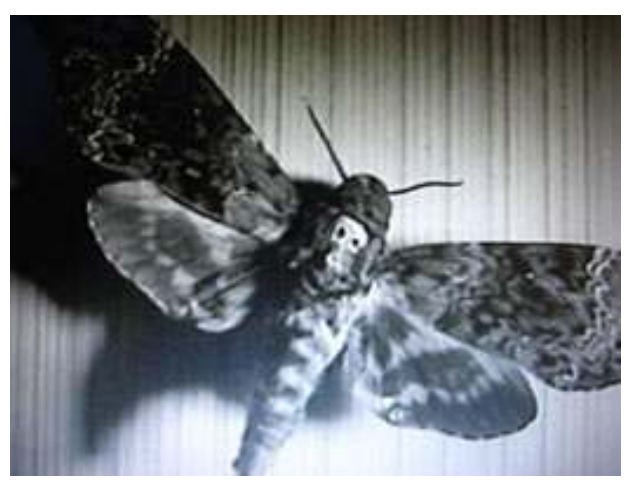
spécimen entomologiste avec le papillon et le crâne. Dans cette perspective, il est une référence commune à W. Benjamin et à W. G. Sebald qui permet de saisir comment la question de l'image se tient à l'articulation de la dialectique entre histoire et nature que ses deux auteurs mettent en place : il s'agit de La Leçon d'anatomie du Docteur Nicolaas Tulp (1632) de Rembrandt. Dans Les Anneaux de Saturne (Die Ringe des Saturns), Sebald reproduit la toile de Rembrandt réalisée en 1632, une fois intégralement, une autre en cadrant sur le centre de la toile où se déroule l'opération d'incision (fig. 1 et 2).

Fig. 1

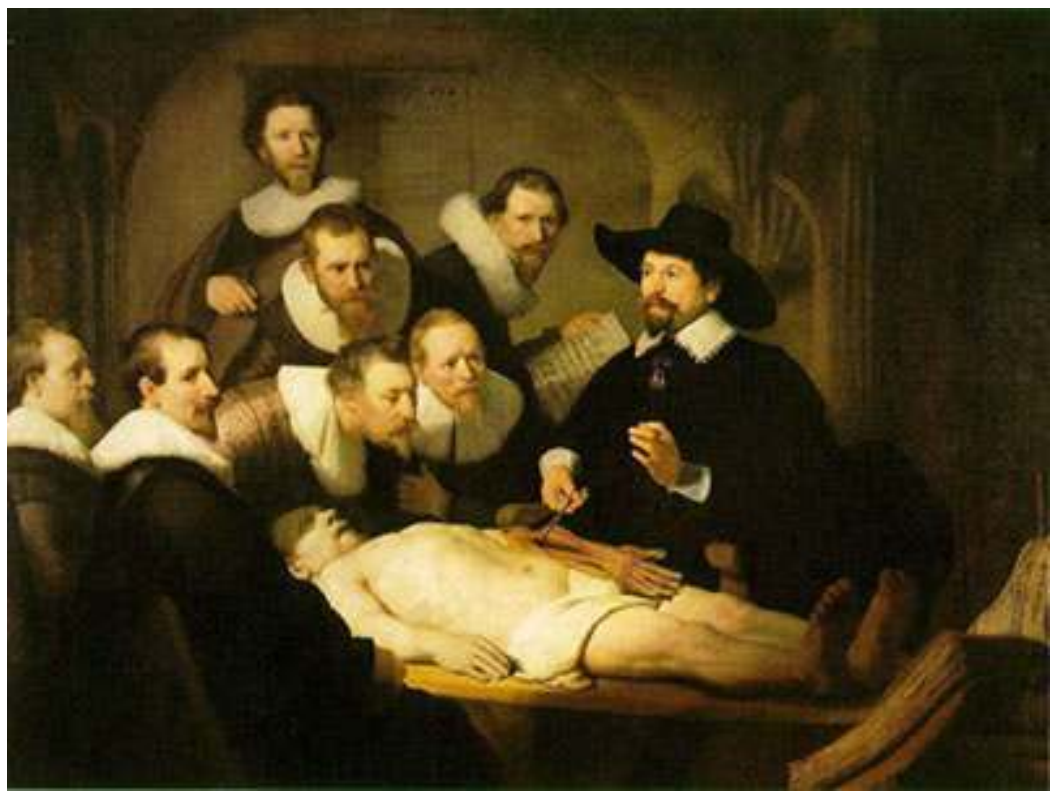

Rembrandt, La Leçon d'anatomie du professeur Tulp, huile sur toile 162×216, Mauritshuis, La Haye. 
Fig. 2

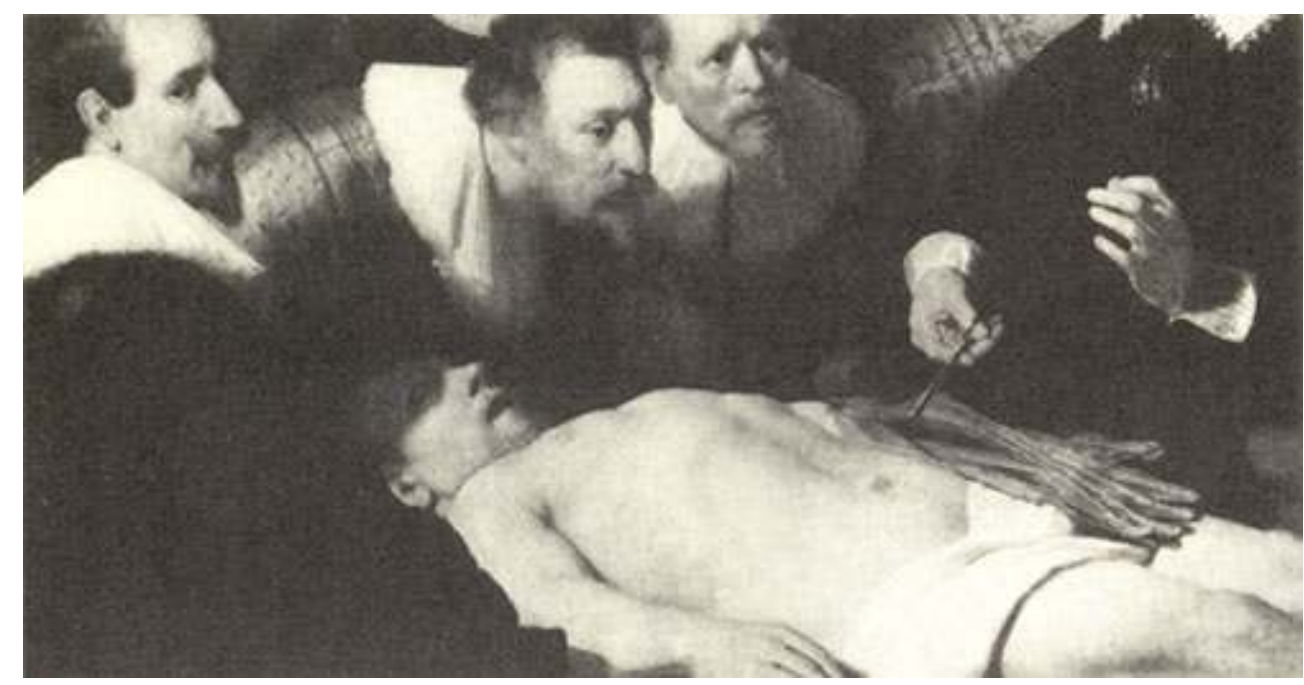

Détail de Rembrandt, La Leçon d'anatomie du professeur Tulp, in W. G. Sebald, Les Anneaux de Saturne, p. 30.

De cette toile, dont il existait un « double », La leçon d'anatomie du Docteur John Deyman de 1656 , détruite dans un incendie en 1723, l'écrivain propose une lecture qui va à l'encontre des interprétations réalistes. Il affirme que la main de la victime, « là où la chair a d'ores et déjà été incisée [est] totalement inversée du point de vue strictement anatomique [puisque] les tendons dénudés qui devraient être ceux de la paume de la main gauche sont en fait ceux du dos de la main droite. Il s'agit donc d'une figure purement scolaire, d'un emprunt à l'atlas d'anatomie $"^{3}$. Il remarque ensuite que les personnages de la guilde des médecins ne regardent pas le cadavre, et que seul le spectateur du tableau est enjoint de l'observer. En particulier là où le corps est anatomisé, sur l'avant-bras gauche d'Aris Kindt - auquel Sebald restitue son identité. Pour l'écrivain, cette toile exhibe un « défaut de construction criant [...] à l'endroit même où s'exprime sa signification centrale »- le lieu de l'incision. Afin d'appuyer son propos, sebald recadre la toile pour nous montrer comment, à l'encontre des interprétations réalistes, le maître a rendu évident un montage entre deux représentations du corps: picturale et scientifique. "Il est à peine pensable que Rembrandt ait fait cela sans le vouloir. ». Dans le choc entre ces deux représentations, « la main difforme témoigne de la violence qui s'exerce à l'encontre d'Aris Kindt. C'est avec lui, avec la victime et non avec la guilde des chirurgiens qui lui a passé commande du tableau, que le peintre s'identifie. Lui seul n'a pas le froid regard cartésien, lui seul perçoit le corps éteint, verdâtre, voit l'ombre dans la bouche entrouverte et sur l'œil du mort $»^{4}$. Tandis qu'aucun des participants de la Leçon ne regarde le corps anatomisé, ce regard empathique du peintre sur la victime devient aussi celui du spectateur dont l'œil est attiré sur l'endroit de cette incision qui dérange les normes du réalisme. Mais, surpris par ce "défaut » que provoque le montage, il est également conduit à prendre une distance critique vis-à-vis de la scène : derrière le noble exercice de la médecine apparaît la violence « du rituel archaïque de démembrement d'un homme $»^{5}$. Avec cette démonstration, Sebald défend un regard qui doit être précis et incisif comme celui de l'anatomiste, mais aussi artistique, capable d'empathie avec ce qu'il regarde, afin de donner la parole à ceux qui ne peuvent plus parler : les disparus, les morts, les victimes de la destruction. Or, dans ces pages, c'est justement en anatomisant l'image grâce au 
cadrage que Sebald propose une analyse inédite de la toile de Rembrandt. Il montre ce qui n'y est pas immédiatement visible : un inconscient collectif qui rejoue un rituel archaïque.

Fig. 3

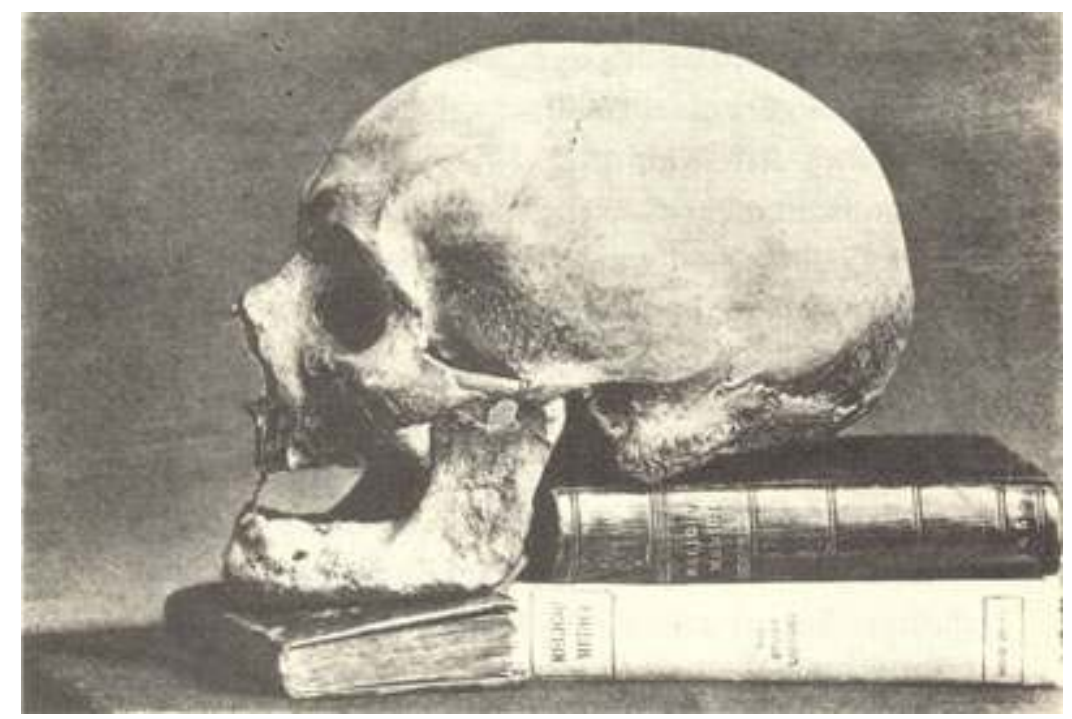

W.G. Sebald, Les Anneaux de Saturne (Die Ringe des Saturns, 1995), trad. de Bernard Kreiss, Arles, Actes Sud, 1999. Folio, 2003, p. 24

Le narrateur est alors sur les traces de Thomas Browne, et plus précisément de son crâne (fig. 3), l'éminent écrivain et médecin ayant probablement été présent à la fameuse leçon représentée par Rembrandt. 
Fig. 4

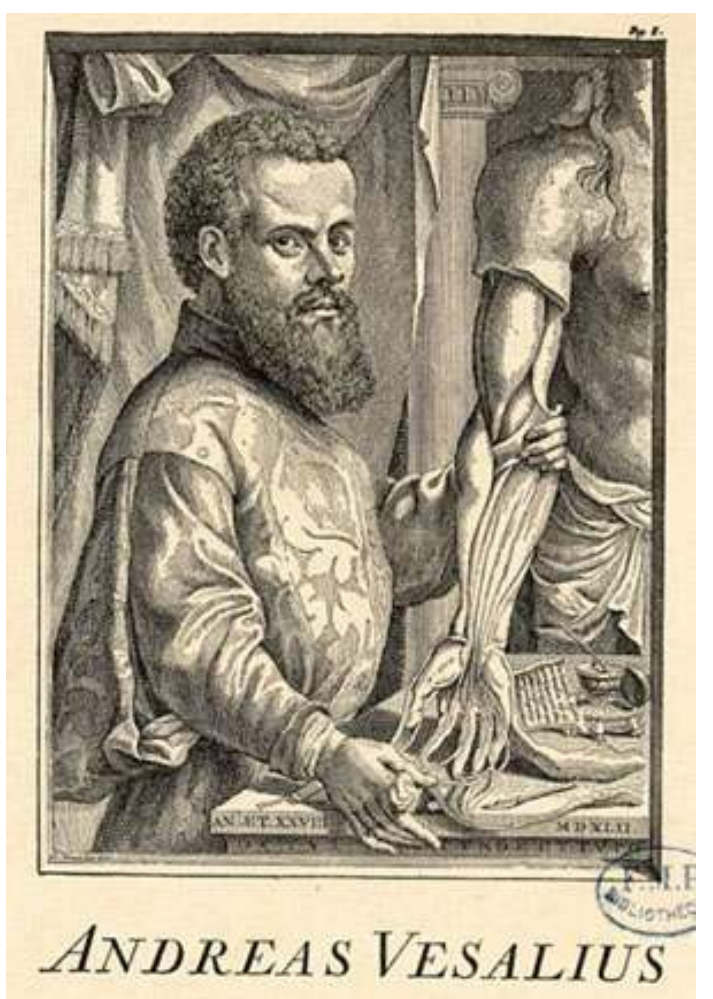

André Vésale, frontispice intérieur de l'ouvrage De corporis humani fabrica libri septem, 1543.

4 Une leçon d'anatomie à partir de Rembrandt dont l'opération heuristique et herméneutique actualise les propositions de W. Benjamin dans L'Euvre d'art à l'époque de sa reproductibilité technique : selon ce dernier, les moyens auxiliaires de la photographie et du cinéma (ralenti, accéléré, gros plan, etc.) font émerger de nouvelles possibilités cognitives offertes. Or, dans la troisième version de cet ouvrage, W. Benjamin fait référence en note à ce tableau alors qu'il examine comment la technique satisfait le désir des masses de rendre spatialement et humainement les choses plus proches de soi. Il s'agit en effet de posséder la chose ou l'ailleurs dans l'image, ou plutôt, dans son reflet, sa reproduction : "Que les choses deviennent "humainement plus proches" des masses, cela peut signifier qu'on ne tient plus compte de leur fonction sociale. Rien ne garantit qu'un portraitiste contemporain, quand il représente un célèbre chirurgien prenant son petit déjeuner entouré de sa famille, saisisse plus exactement sa fonction sociale qu'un peintre du seizième siècle [sic] qui, comme le Rembrandt de la Leçon d'anatomie, présentait au public de son temps une haute image des médecins ${ }^{6}$. Cette remarque, ajoutée en note dans la version de 1935, annonce la métaphore de l'anatomie qui arrive peu après dans le texte et replace la toile dans la tradition iconographique du portrait du chirurgien. Rembrandt en effet se réfère pour son œuvre au portrait d'André Vésale (fig. 4) présenté en frontispice du premier ouvrage illustré du genre, publié en 1543, De corporis humani fabrica libri septem, qui est certainement celui que tient entre les mains l'un des spectateurs de l'opération.

Or, à regarder ces deux portraits de chirurgien, où l'un est en représentation (en leçon) tandis que l'autre figure à lui seul le geste anatomique, on constate que l'opération ellemême diffère : là où Vésale empoigne l'avant-bras disséqué du cadavre ${ }^{7}$, Tulp s'en saisit et l'exhibe grâce à une pince chirurgicale qui est venue relayer le scalpel. En un siècle, 
l'instrument vient médiatiser la pénétration du corps. Le portrait de Tulp se démarque en effet de celui de son prédécesseur car il ne plonge pas ses mains dans le cadavre mais recourt à un instrument. Dans cette peinture, comme dans la comparaison entre le chirurgien et le cameraman chez W. Benjamin, ce qui prime c'est l'instrument ou «l'appareil ». W. Benjamin ne manque alors pas de souligner la spécificité paradoxale de la camera comme de l'instrument anatomique : «Le chirurgien, à l'instant décisif, renonce à s'installer en face du malade dans une relation d'homme à homme; c'est plutôt opérativement qu'il pénètre en lui. [...] Pour l'homme d'aujourd'hui, l'image du réel est incomparablement plus significative, car, si elle atteint à cet aspect des choses qui échappe à tout appareil et que l'homme est en droit d'attendre de l'œuvre d'art, elle n'y réussit justement que parce qu'elle use d'appareils, pour pénétrer, de la façon la plus intensive, au cœur même de ce réel. [...] La réalité est ici la plus artificielle que l'on puisse imaginer et, au pays de la technique, le spectacle de la réalité immédiate s'est transformé en fleur bleue introuvable $»^{8}$. Alors même que le cameraman appréhende la réalité par la médiation d'un appareil, il se tient au plus près de celle-ci, et la restitue au spectateur touché dans son intimité. L'image intervient ainsi au niveau de «l'historicité de la biographie individuelle " justement parce qu'elle rend compte avec une précision anatomique de "la nature de l'existence humaine $»^{9}$. De son côté, le chirurgien pénètre au plus profond de la chair en conservant avec elle la distance que lui permet son instrument. Il découvre ainsi l'historicité d'un corps particulier, les singularités de ses organes, mais révèle également des invariants universels propres au corps humain. Pour W. Benjamin, ce paradoxe de l'opération anatomique qui dialectise le proche et le lointain de l'intime et de l'universel, dont il fait l'attribut de l'image cinématographique (mais aussi photographique) et qu'il explicite grâce à la comparaison avec le chirurgien et la métaphore de l'opération anatomiste, confère à l'image produite par la modernité sa dimension allégorique. Siegfried Kracauer le note, en 1940, alors qu'il est en transit avec son ami W. Benjamin à Marseille : « La face dans le film n'a pas de valeur si elle ne fait pas affleurer la tête de mort qui la sous-tend. Danse macabre. Mais à quelle fin ? Cela reste à voir $»^{10}$.

\section{Le regard anatomiste : un dispositif heuristique}

Si Theodor W. Adorno et Max Horkheimer stigmatisent la technique comme ce qui condamne l'art au mécanisme et à la culture de masse ${ }^{11}$, W. Benjamin l'appréhende comme un "nouveau concept $»^{12}$ grâce auquel la modernité apprend à voir. Avec la métaphore du regard de l'anatomiste, W. Benjamin définit l'œil appareillé comme un dispositif heuristique: le caractère incisif de la science anatomique délivre une vision inédite du corps tout comme le regard photographique et cinématographique offre une visibilité inattendue du réel. La métaphore apparaît dans L'Euvre d'art à l'époque de sa reproductibilité technique comme dans la Petite Histoire de la photographie ${ }^{13}$ et s'applique à l'image photographique et cinématographique. 
Fig. 5

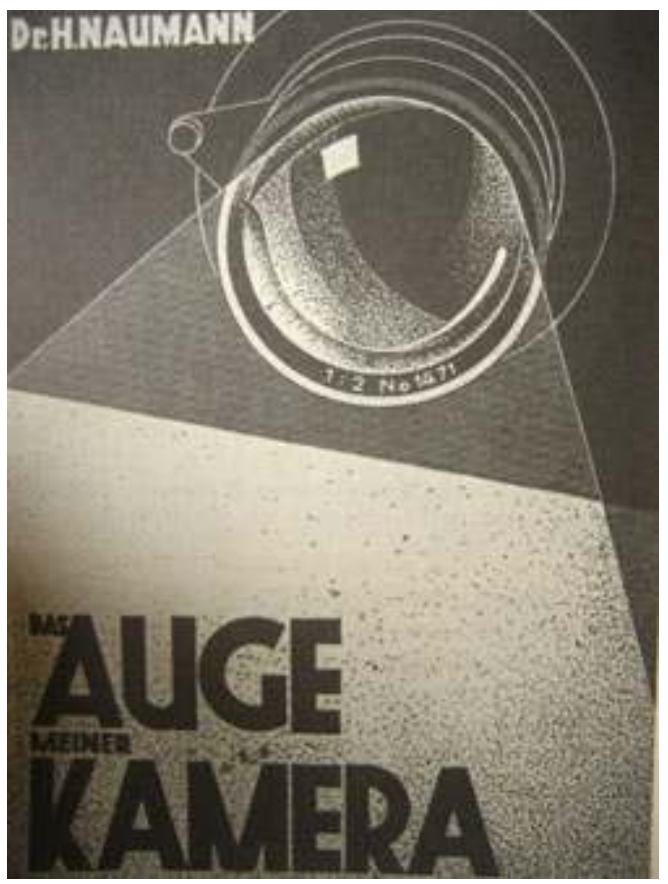

Helmut Neumann, L'EFil de ma caméra (Das Auge meiner Kamera. Eine Buch über fotografische Optik), Halle/Saale, 1037.

Fig. 6

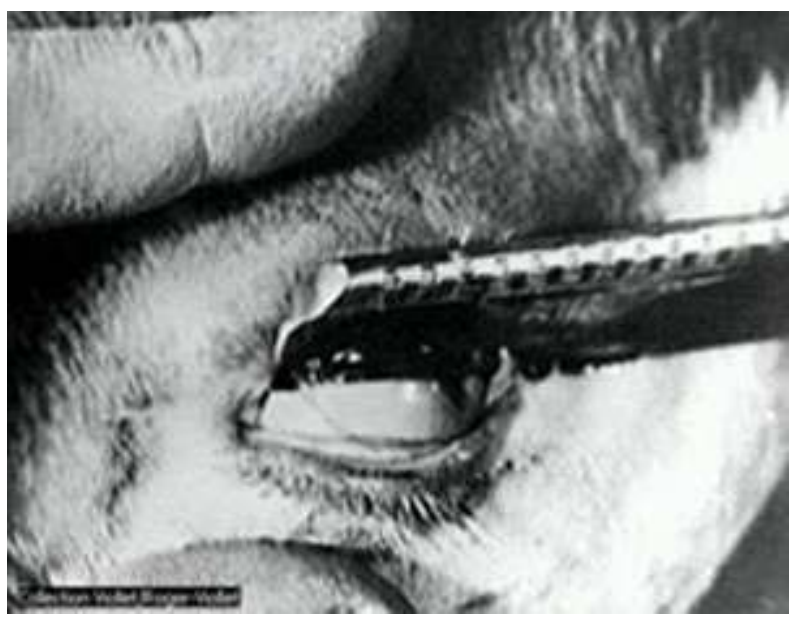

Luis Buñuel et Salvador Dali, Un chien andalou, 1929.

7 Dès la première version de l'essai sur l'invention de la reproduction mécanisée et ses effets sur l'art, la métaphore du regard anatomiste apparaît à la suite d'une comparaison entre "caméraman" et "chirurgien». Elle vient à l'appui d'une démonstration qui vise à établir la différence entre la représentation de la réalité par le cinéma et le théâtre, puis la peinture. Elle est tirée de «l'idée même d'opération », W. Benjamin désignant le cameraman comme un «opérateur » (der Operateur), « celui qui fait l'œuvre» cinématographique, l'opera, mais aussi «qui fait fonctionner un appareil ». Grâce à l'œil de la caméra (fig. 5), l'opérateur « pénètre en profondeur dans 
la trame du donné $»^{14}$ : il fait apparaître, met au jour, il tranche à vif, et en premier l'œil du spectateur comme celui de la jeune femme dans Un chien andalou (fig. 6).

Fig. 7

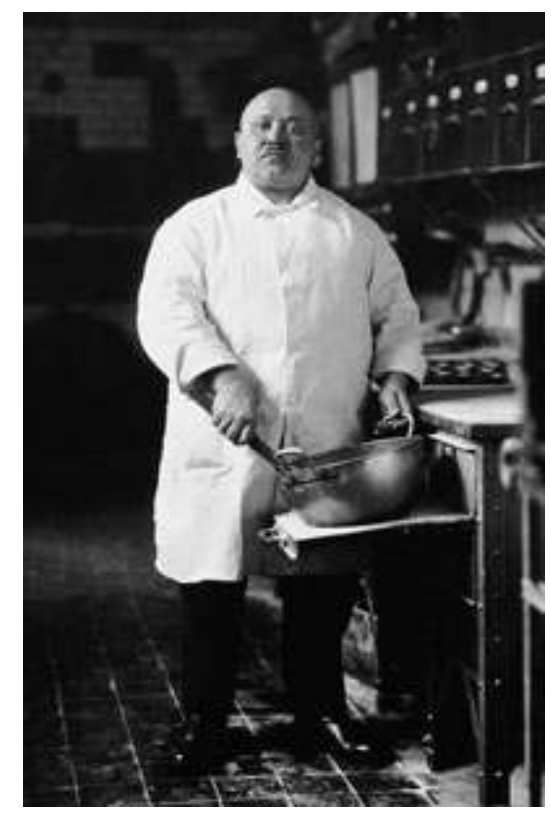

August Sander, « Le pâtissier », Visage d'une époque (Das Antlitz der Zeit), 1928.

Surréaliste ici parce qu'incarnant la volonté politique de briser les apparences et le vernis d'un art bourgeois, ce regard va du manifeste au caché; il confine à une opération de déchiffrement du réel, celui-ci se sclérosant dans la maladie sociale. La métaphore du regard de l'anatomiste est récurrente dans l'entre-deux Guerres. Durant cette période de misère économique et de manifestes, la volonté de voir ce qui est en train d'advenir détermine sa résurgence dans les discours. On la retrouve chez $\mathrm{B}$. Brecht, mais aussi chez un partisan d'un tout autre bord politique, le médecin français Céline $^{15}$. Garante d'une entreprise salutaire de déchiffrement de la maladie sociale, cette métaphore sert divers bords politiques; elle caractérise le regard lucide de l'artiste face à la crise sociale et culturelle que le progrès technique a établie. Bertold Brecht, qui avait fait des études de médecine, compare le médecin et l'écrivain, l'un et l'autre déterminant la cause exacte de la maladie, livrant un diagnostique ${ }^{16}$. Son compatriote, le médecin Alfred Döblin, cité par W. Benjamin dans sa Petite histoire de la photographie, écrit dans sa préface à l'ouvrage du photographe August Sander publié en 1929, Antlitz der Zeit (Visage d'une époque) : «De même qu'il existe une anatomie comparée, éclairant notre compréhension de la nature et de l'histoire de nos organes, de même Sander nous propose-t-il la photographie comparée: une photographie dépassant le détail pour se placer dans une perspective scientifique $»^{17}$. Les clichés de Sander (fig. 7) sont une "observation directe " de la réalité, un regard objectif qui anatomise les visages pour découvrir leur provenance sociologique. 
Fig. 8

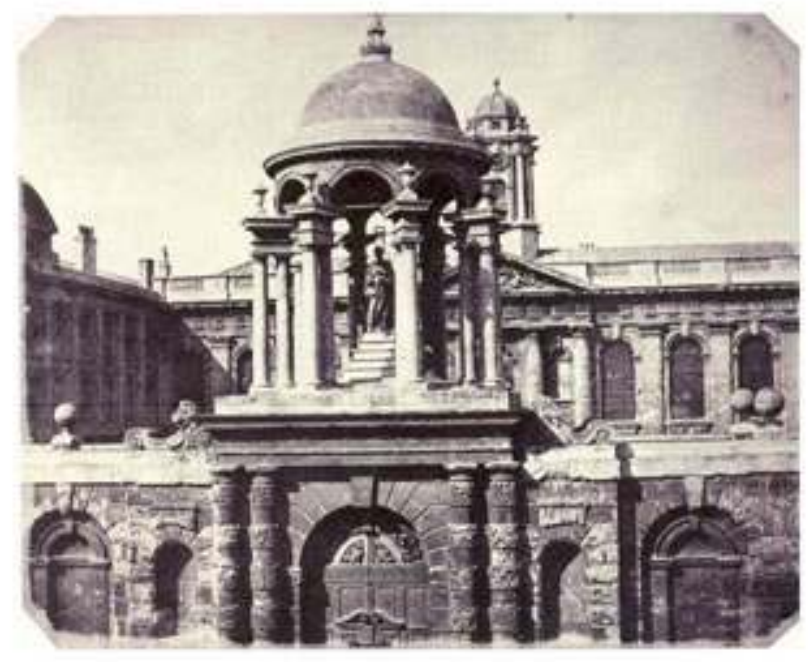

W. H. F. Talbot, «Le portail d'entrée de Queen's College », Planche XIII, Le Pinceau de la nature (The Pencil of Nature), 1844.

Fig. 9

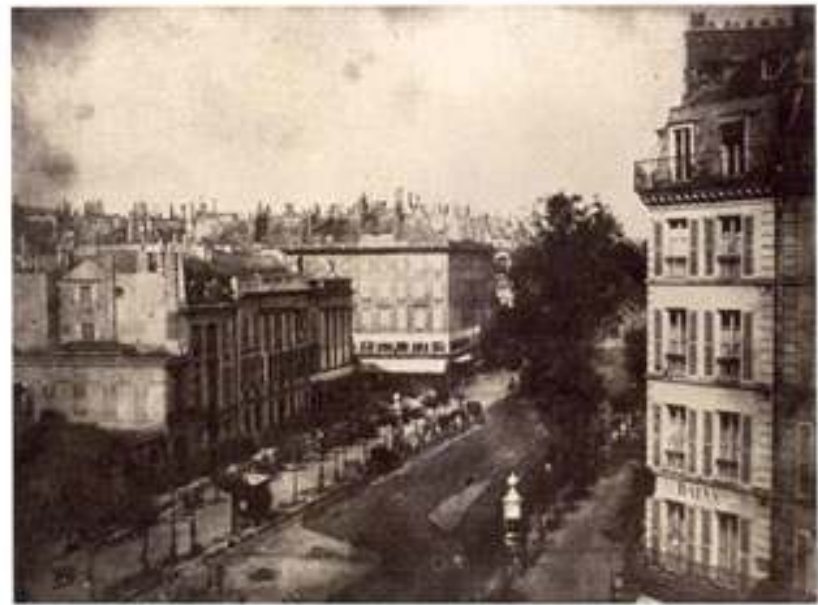

W. H. F. Talbot, « Paris, vue sur les boulevard», Planche II, Le Pinceau de la nature (The Pencil of Nature), 1844.

9 La photographie (et le cinéma) démasque la réalité, révèle les identités, surprend la nature dans des détails jusqu'alors invisibles. C'est ce que montrait déjà admirablement l'inventeur du calotype, W. H. F. Talbot, dans son Pencil of Nature publié en 1844. Précurseur de la modernité avec son ouvrage qui, le premier, allie le texte et la photographie grâce à un savant montage entre ces mediums, Talbot aide son lecteur (dans la planche II [fig. 8]) à comprendre la raison de traces sombres sur le sol : « L'air est chaud et poussiéreux et la chaussée vient d'être arrosée ». 
10 Le spectateur découvre la mémoire latente de l'image, certains éléments de celle-ci pouvant avoir échappé au photographe lui-même, comme « un cadran dans le lointain, sur lequel, inconsciemment (unconsciously), on a enregistré l'heure de la prise de vue ${ }^{18}$ (fig. 9).

Fig. 10

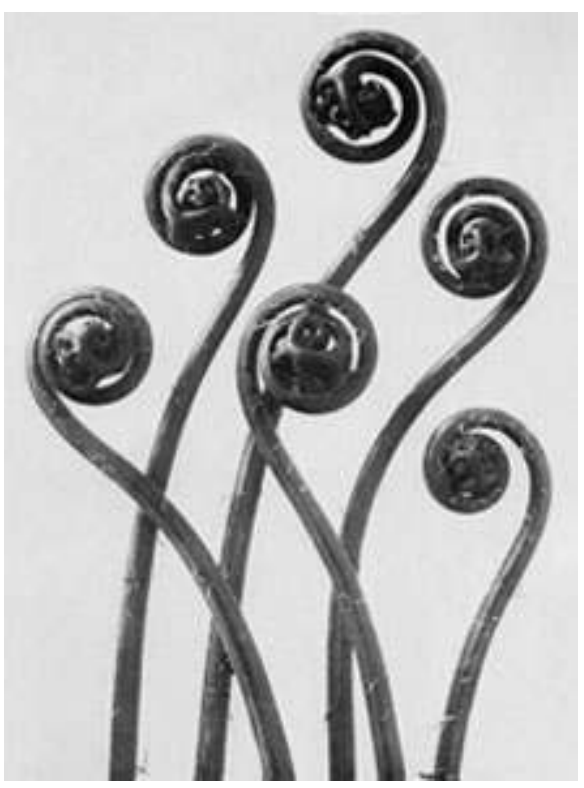

Karl Blossfeld, « Haarfarn », Les Formes originelles de l'art (Urformen der Kunst), 1928.

11 Un demi-siècle plus tard, l'objectif n'est plus un crayon ou un pinceau qui saisit de la nature les moindres détails mais un «scalpel » qui nous fait pénétrer « de la façon la plus intensive, au cœur même $[\mathrm{du}]$ réel $»^{19}$. W. Benjamin décrit ainsi un véritable dispositif heuristique rendu possible grâce aux moyens auxiliaires de la caméra, c'est-àdire «ses plongées et ses remontées, ses coupures et ses isolements, ses ralentissements et ses accélérations du mouvement, ses agrandissements et ses réductions ». Ainsi, le cinéma tout comme la photographie «nous renseigne[nt] sur cet inconscient visuel, comme la psychanalyse sur l'inconscient pulsionnel $»^{20}$. Dans La Petite histoire de la photographie, W. Benjamin donne en exemple les fameuses images de fleurs de Karl Blossfeld qui, grâce à l'agrandissement et au cadrage, révèlent dans une arborescence de fougère " une crosse épiscopale " ${ }^{21}$ (fig. 10).

12 Ainsi, la modernité rejoue la double dimension artistique et scientifique de l'observation de la nature : « En effet, lorsqu'on considère un comportement en l'isolant bien proprement à l'intérieur d'une situation déterminée - comme on découpe un muscle dans un corps - on ne peut plus guère savoir ce qui nous fascine le plus : sa valeur artistique ou son utilité pour la science ${ }^{22}$. La métaphore du regard anatomiste ressortit à une tradition où la science et l'art échangent leurs pratiques. Dès le début du $\mathrm{XV}^{\mathrm{e}}$ siècle, les médecins, pour des raisons didactiques notamment, ont eu recours à l'image pour expliciter et fonder leur découverte. À l'inverse, les artistes en appellent au savoir anatomique pour rendre leur art plus adéquat à l'impératif de la mimesis ${ }^{23}$. La double qualité scientifique et artistique que W. Benjamin attribue à la photographie et au cinéma est au cœur d'une conception de la beauté où celle-ci ne dure que d'être objet de savoir : «il n'y a pas de beauté, s'il n'y a pas au plus profond d'elle-même un 
quelconque objet de savoir $»^{24}$. W. Benjamin apporte ces précisions historiques sur l'anatomie dans les notes de bas de pages de la dernière version de L'Euvre d'art, dans lesquelles il fait également référence à Léonard de Vinci. Si ces notes de bas de pages historicisent la métaphore, elles l'inscrivent également dans une histoire naturelle dont elle redéfinit les enjeux épistémologiques : comme jadis l'exercice de dissection a fait progresser notre connaissance du corps humain, les instruments de la modernité que sont l'objectif et la caméra offrent de nouvelles possibilités cognitives. L'une et l'autre transforment les modalités de la création comme de l'observation, et relèvent à la fois de l'histoire de l'art et de l'histoire naturelle.

\section{L'image papillon : un dispositif herméneutique}

13 L'enjeu de cette naissance du regard moderne est de taille puisqu'il consacre une nouvelle lisibilité du monde dans les images et la découverte d'un point de vue différent sur la nature : « La nature qui parle à l'appareil photographique est autre que celle qui parle à l'œil - autre, avant tout, en ce qu'un espace consciemment travaillé par l'homme se substitue un espace élaboré de manière inconsciente ${ }^{25}$ ». En 1930, Jean Epstein décrit également cette Photogénie de l'impondérable que dévoile la photographie et le cinéma: "Le ralenti et l'accéléré révèlent un monde où il n'y a plus de frontières entre les règnes de la nature. Tout vit. Les cristaux grandissent, vont au devant les uns des autres, se joignent avec les douceurs de la sympathie. Les symétries sont leurs mœurs et leurs traditions. Qu'ont-ils de si différents des fleurs ou des cellules de nos plus nobles tissus ? Et la plante qui dresse sa tige, qui tourne ses feuilles vers la lumière, qui étale et clôt sa corolle, qui incline son étamine sur le pistil, n'a-t-elle pas, à l'accéléré, exactement la même qualité de vie que ce cheval et son cavalier qui, au ralenti, planent au-dessus de l'obstacle et s'inclinent l'un sur l'autre. Et la pourriture est une renaissance ${ }^{26}$. Ce que la caméra saisit et rend visible c'est «la qualité de la vie», comme le dit bien Epstein, son mouvement. La technique permet ainsi d'exaucer Goethe préconisant d'observer la nature vivante: «Le pauvre animal palpite dans les filets et perd en se débattant ses plus belles couleurs ; et même si on réussit à l'attraper intact, le voilà quand même pour finir épinglé là, rigide, et sans vie; le cadavre n'est pas la totalité de l'animal, quelque chose d'autre en fait partie, partie principale, et en cette occurrence comme en tout autre, partie principale des principales : la vie ${ }^{27}$. Partout chez W. Benjamin affleurent, à la mesure de son époque, des remarques allant dans le sens d'un renoncement à l'approche cognitive des naturalistes. La critique d'une histoire qui suit le modèle positiviste et dont le darwinisme conduit à un capitalisme progressiste culmine dans les thèses Sur le concept d'histoire, s'impose dans la section « Théorie de la connaissance » du Livre des passages et guide une appréhension générale du monde. Ainsi, le papillon, proie favorite de l'entomologiste, devient chez W. Benjamin l'objet d'une chasse qui tourne à l'empathie :

"Quand ainsi une vanesse ou un sphinx de troène, que j'aurais pu tranquillement dépasser, me bernait par ses hésitations, ses oscillations et ses arrêts, j'aurais alors aimé me dissoudre en air et en lumière, simplement pour pouvoir m'approcher inaperçu de ma proie et m'en emparer. Et mon désir se réalisait en ceci que chaque battement ou chaque balancement de ces ailes qui me fascinaient m'effleurait d'un souffle ou d'un frisson. La vieille loi de la vénerie commençait à régner entre nous : plus je me conformais de toutes mes fibres à l'animal, plus je devenais en moi-même lépidoptère, et plus les faits et gestes de ce papillon prenaient la couleur de la 
décision humaine, et, finalement c'était comme si sa prise était le prix que je devais payer pour pouvoir recouvrer ma nature humaine. $»^{28}$

Fig. 11

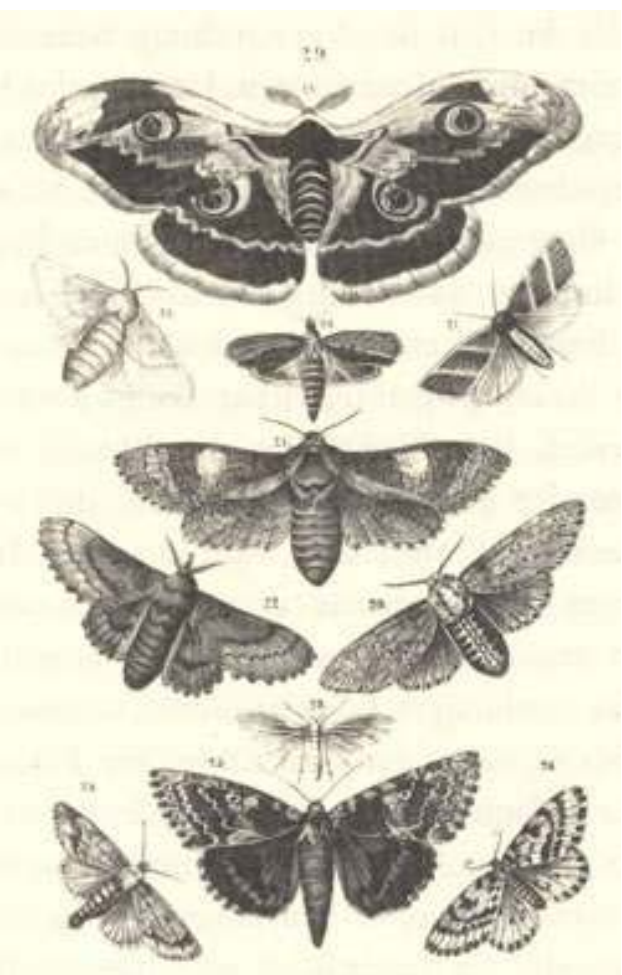

W. G. Sebald, Les Anneaux de Saturne, p. 355 une réserve essentielle vis-à-vis de cette méthode lorsqu'elle touche à l'observation du passé. C'est ce qu'il explique dans les thèses Sur le concept d'histoire où il observe que pour revivre le passé, l'historiciste ne sait s'identifier qu'aux vainqueurs ${ }^{29}$. Or il n'est d'histoire chez W. Benjamin sans traces, sans un matérialisme qui, comme chez S. Kracauer, donne voix aux opprimés, aux victimes, aux laissés-pour-compte de l'histoire. L'historien chiffonnier ne veut pas s'approprier des «formules spirituelles » mais des documents-rebuts, «les guenilles », afin de "leur rendre justice de la seule façon possible : en les utilisant $»^{30}$. Depuis cette marge, une vérité demeurée invisible, un point de vue inédit sur le passé s'impose. La trace, vis-à-vis de laquelle il faut adopter une posture méthodologique adéquate, est semblable au papillon: car il ne s'agit pas d'épingler la trace afin de l'observer scrupuleusement, mais de l'observer vivante, c'est-à-dire en ce qu'elle suscite une expérience de remémoration. Si cette comparaison est une métaphore latente dans le texte de W. Benjamin, elle est manifeste chez W.G. Sebald qui décline le paradigme de l'imago dans ses récits - de l'insecte à l'image ${ }^{31}$ en passant par la réalité psychique - où, en quête de leur passé, les personnages sont des «butterfly men». Avec Les Anneaux de Saturne, le récit suit le fil de soie de la mémoire à partir d'une planche d'entomologie représentant le Bombyx mori (fig. 11) dont la chenille produit la précieuse matière.

15 C'est donc à l'infime, au plus petit, au lépidoptère fragile que s'identifie l'enfant de la chasse aux papillons de W. Benjamin, phalène qui, chez Virginia Woolf déjà, figure la 
condition humaine : «Cet effort gigantesque d'une petite phalène insignifiante pour s'opposer à une puissance aussi démesurée [la mort], afin de conserver ce qui n'avait de valeur pour personne, n'intéressait personne. [...] Comme je contemplais la phalène morte, le triomphe obscur et minuscule d'une si grande force sur un si piètre adversaire m'emplit de stupeur $\aleph^{32}$. Grâce à cet animal banal, enfantin, aux noms pourtant énigmatiques dans le vocabulaire scientifique, s'impose une méditation sur la «nature humaine ». La légèreté du papillon, physique et symbolique, devient gravité méditative.

16 Mais avec la photographie et le cinéma, l'empathie dont il est question est spécifique : il s'agit d'une empathie médiatisée par l'appareil. Le spectateur n'a "aucun contact personnel avec l'interprète. Il n'a de relation empathique avec lui qu'en ayant une relation de ce type avec l'appareil ${ }^{33} »$. Avec cette empathie à l'appareil, W. Benjamin éprouve dans le spectacle des arts la notion de «fantasmagorie » telle que la définit Karl Marx dans Le Capital. D'une part comme un rapport entre objets se substituant à un rapport entre personnes, et procédant à la réification de l'humain en raison de la transformation de la force de travail en marchandise, d'autre part comme un retour du refoulé, le caractère social de la production revenant sous la forme du caractère fétiche de la marchandise, considérée comme source autonome de valeur à travers l'échange et, par là, investie de tous les affects. "Cette âme de la marchandise que Marx mentionne à l'occasion, en plaisantant" consacre "l'empathie avec l'inorganique $»^{34}$ et, pour W. Benjamin, caractérise une manière de regarder la réalité qui, propre à la modernité, révolutionne à la fois le domaine de la science et celui de l'art. Grâce à l'appareil, le regard garde une distance nécessaire tout en s'impliquant dans l'observation de son objet - pareil regard évoque celui du psychanalyste sur son patient avec, à la clef, la notion de transfert élaborée par Freud : c'est un dispositif herméneutique fondé sur la dialectique du clinique et de l'empathique. Car, avec les techniques du cinéma et de la photographie, il est désormais possible d'anatomiser le réel.

17 Entre la métaphore de l'anatomie et celle du papillon, les images naturalistes viennent donc définir les possibilités cognitives d'un nouveau regard sur la réalité et le passé, regard indéniablement mélancolique ${ }^{35}$. C'est en premier lieu grâce à ces métaphores que «la transformation de l'histoire en nature » chez W. Benjamin est lisible : elles formalisent une «intention allégorique $»^{36}$ qui vise à rendre compte du rythme implacable de la destruction.

\section{Face au crâne : le dispositif allégorique}

18 Avec l'Origine du drame baroque allemand, $\mathrm{W}$. Benjamin définit le statut moderne de l'allégorie en lien avec «la contemplation en larmes» du mélancolique. «Surgi[e] du fond de l'abîme de la méditation ", elle se distingue du symbole qui relève de la temporalité transcendantale des fins dernières et du salut, alors que l'allégorie est inscrite dans la temporalité de l'histoire naturelle :

«Alors que dans le symbole, par la sublimation de la chute, le visage transfiguré de la nature se révèle fugitivement dans la lumière du salut, en revanche dans l'allégorie, c'est la facies hippocratica de l'histoire qui s'offre au regard du spectateur comme un paysage primitif pétrifié. L'histoire, dans ce qu'elle a toujours eu d'intempestif, de douloureux, d'imparfait, s'inscrit dans un visage - non : dans une tête de mort. Et aussi vrai qu'il n'y a en celle-ci nulle liberté "symbolique", nulle harmonie classique de la forme, nulle humanité, l'énigme qui s'exprime dans 
cette figure, la plus soumise à la nature, ce n'est pas simplement la nature de l'existence humaine, mais l'historicité de la biographie individuelle. C'est là le noyau de la vision allégorique, de l'exposition baroque de l'histoire comme histoire des souffrances; elle n'a de signification que dans les stations de sa décadence. Autant de sens, autant d'emprise de la mort, parce que la mort enfouit au plus profond la ligne de démarcation brisée qui sépare la physis et la signification. Mais si la nature a de tout temps été gouvernée par la mort, elle a toujours été allégorique. $»^{37}$

La puissance de la physis est d'abolir la signification. Le cours des choses ne fait plus sens, il n'est plus que continuité sans fin de la destruction.Avec l'allégorie, l'histoire révèle son véritable visage, une tête de mort : elle n'a point de salut à l'horizon, point de finalité transcendantale, elle ne progresse que de destruction en destruction, soumise à l'histoire de la nature. C'est une "histoire des souffrances" qui n'a de «signification que dans les stations de la décadence »: « l'histoire n'est pas modelée, figurée comme le processus d'une vie éternelle, mais bien plutôt comme celui d'un destin inéluctable $»^{38}$. T. W. Adorno se consacre, dans un texte de 1932, à cette "idée de l'histoire naturelle ».Chez W. Benjamin, selon lui, ce destin inéluctable est un chiffre, une allégorie qui donne à lire la superposition dialectique de la nature et de l'histoire ${ }^{39}$. Elle figure une vie dépourvue de toute référence sécurisante à la transcendance et totalement exposée au rythme implacable de l'histoire naturelle. Car l'histoire n'est qu'un espace en ruine, là traînent, après la bataille, les restes de la destruction. Cette vision allégorique d'une histoire-tête de mort déjoue toute interprétation transcendantale du passé, échappe au stéréotype eschatologique, et se soumet à la nature. La vie, parmi les monceaux de gravats, reprend déjà ses droits avec la faune et la flore, et les ruines sont arrachées des mains de l'histoire par une histoire naturelle qui impose la réalité du monde dans le continuum de ses calamités: "On pouvait déterminer la date de la destruction en fonction de la végétation, c'était une question de botanique $\aleph^{40}$. Mais il ne s'agit pas seulement d'une naturalisation de l'historique, car si la nature recouvre les ruines de l'histoire, cette dernière de nouveau la détruit. Il s'agit plutôt d'une dialectique sans fin où histoire et nature n'en finissent pas de périr. 
Fig. 12

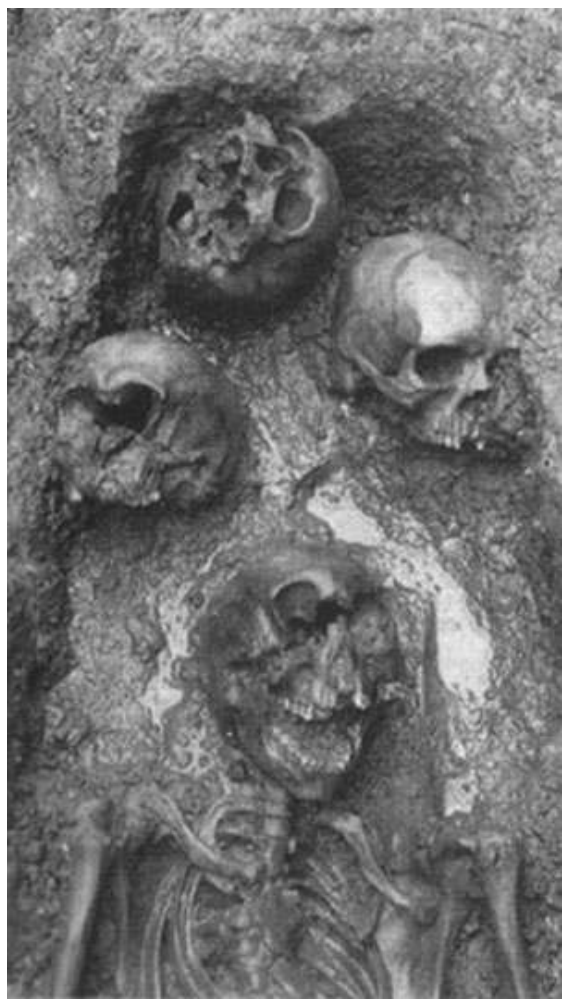

W. G. Sebald, Austerlitz (2001), trad. Patrick Charbonneau, Austerlitz, Arles, Actes Sud, 2002. Folio, 2006, p. 183.

De cette vision allégorique d'une histoire-tête de mort, W. G. Sebald offre un exemple lorsqu'il décrit les strates temporelles se trouvant sous la gare de Liverpool Street Station dans Austerlitz ${ }^{41}$. Au terme de cette exploration archéologique apparait l'image de crânes découverts par les archéologues avec lesquels discute JacquesAusterlitz (fig. 12). 
Fig. 13

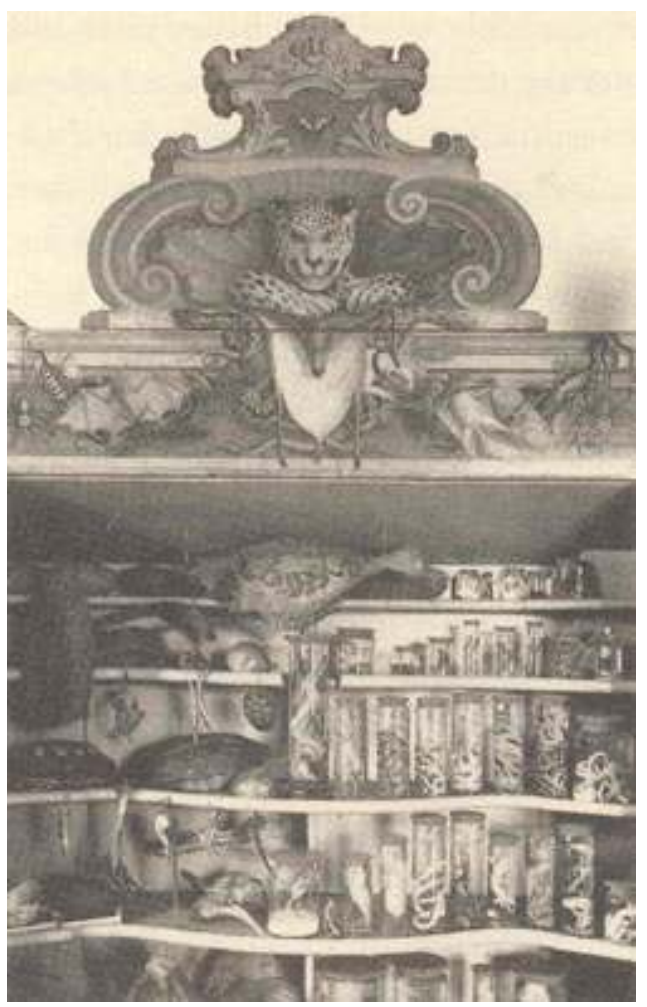

W.G Sebald, Séjours à la campagne (Logis im Landhaus, 1998), trad. Patrick Charbonneau, Arles, Actes Sud, 2006, p. 108.

21 Elle s'impose comme la vision allégorique résultant d'un long processus historique et face à laquelle, l'homme connaît le memento mori. Car rien ne ressemble à un crâne comme un autre crâne, rien n'inscrit mieux la singularité d'une biographie - ce crâne, le mien - dans un destin universel et anonyme. Cette valeur allégorique du crâne est portée par la tradition picturale des Vanités. Et si le paysage historique des ruines devient une nature morte chez W. Benjamin, les cabinets d'histoire naturelle s'imposent aussi comme des visions allégoriques, notamment chez W.G. Sebald (fig. 13).

Car, dans l'opération qui consiste à épingler la vie pour l'observer morte se joue la césure entre la physis et la signification à l'origine du choc du memento mori. La nature scrupuleusement observée perd sa signification véritable, la vie, et devient, à proprement parler une nature morte - en l'occurrence " avec papillon » selon un topos $\mathrm{du}$ genre pictural en question. Planches de botanique, atlas d'anatomie ou de géographie reproduisent la vie comme un schéma ou une mécanique. Au revers de l'expérimentation scientifique se tient alors une vision allégorique qui convie le regard à la méditation immobile, au temps suspendu d'une nature morte ou d'une photographie de ruines. 
Fig. 14

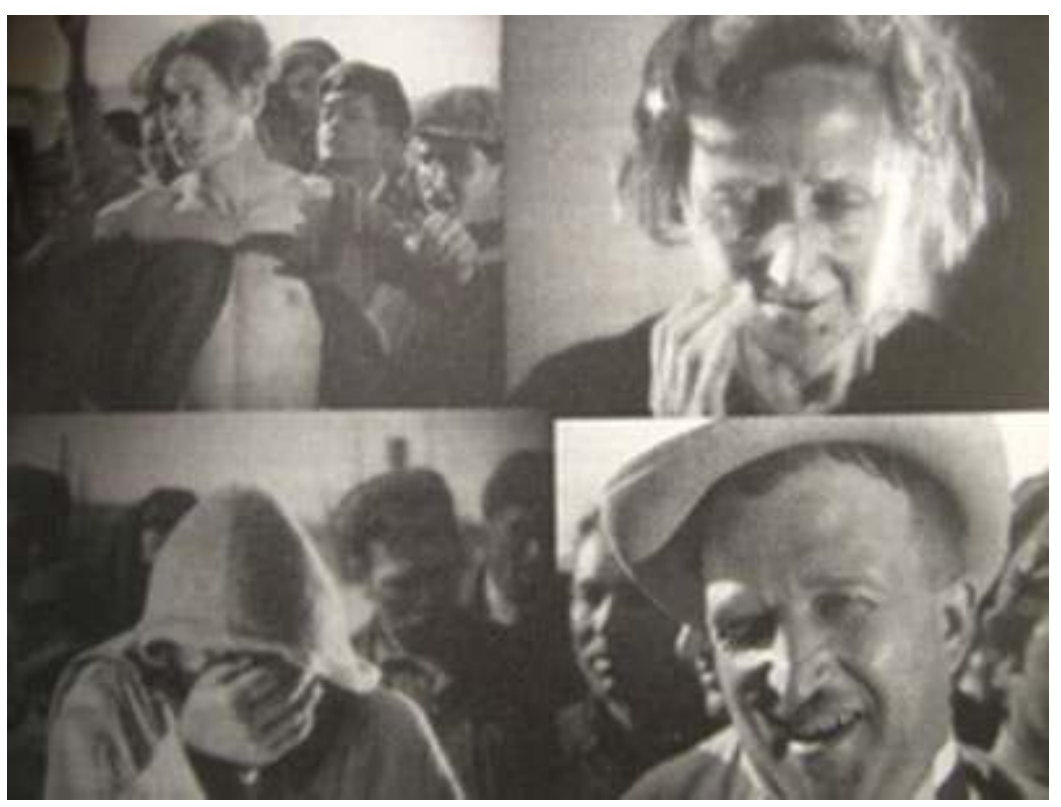

Sergueï Eisenstein, Le Cuirassé Potemkine, 1925.

Ainsi, au milieu des décombres, «le méditatif dont le regard, horrifié, tombe sur le fragment qui est dans sa main, devient allégoricien. ${ }^{42}$ » Cette prise de position face à la destruction et le destin inéluctable qu'elle représente conditionne l'approche micrologique de Benjamin - et, partant de là, sa proposition méthodologique du montage.Le fragment est le matériau de l'historien matérialiste, allégoricien moderne qui renoue avec la vision baroque et mélancolique du monde comme accumulation de rebuts, de restes, d'objets morts, épars autour de l'ange de Dürer. «Le rapport de l'allégorique avec tout ce qui est fragmentaire ${ }^{43}$ s'inscrit chez W. Benjamin dans une appréhension monadologique de la connaissance : «l'idée est monade » ${ }^{44}$, à savoir que chaque idée est une image du monde, le plus petit reflétant la totalité. La technique, chez W. Benjamin, permet de rejouer le topos de la fragmentation allégorique, comme le montre Sigrid Weigel ${ }^{45}$, et caractérise le regard d'une philosophie critique qui cherche «à découvrir dans l'analyse du petit moment singulier le cristal de l'événement total $»^{46}$. Or, ce que va systématiser la modernité et, en particulier, la photographie et de cinéma, c'est le montage. Si pour W. Benjamin, «le montage véritable part du document ", c'est qu'il est moins un instrument de la fiction que celui d'une écriture de l'histoire dans les images et en marge de l'historiographie - ce que montre bien la production cinématographique de Serguei Eisenstein (fig. 14). 
Fig. 15

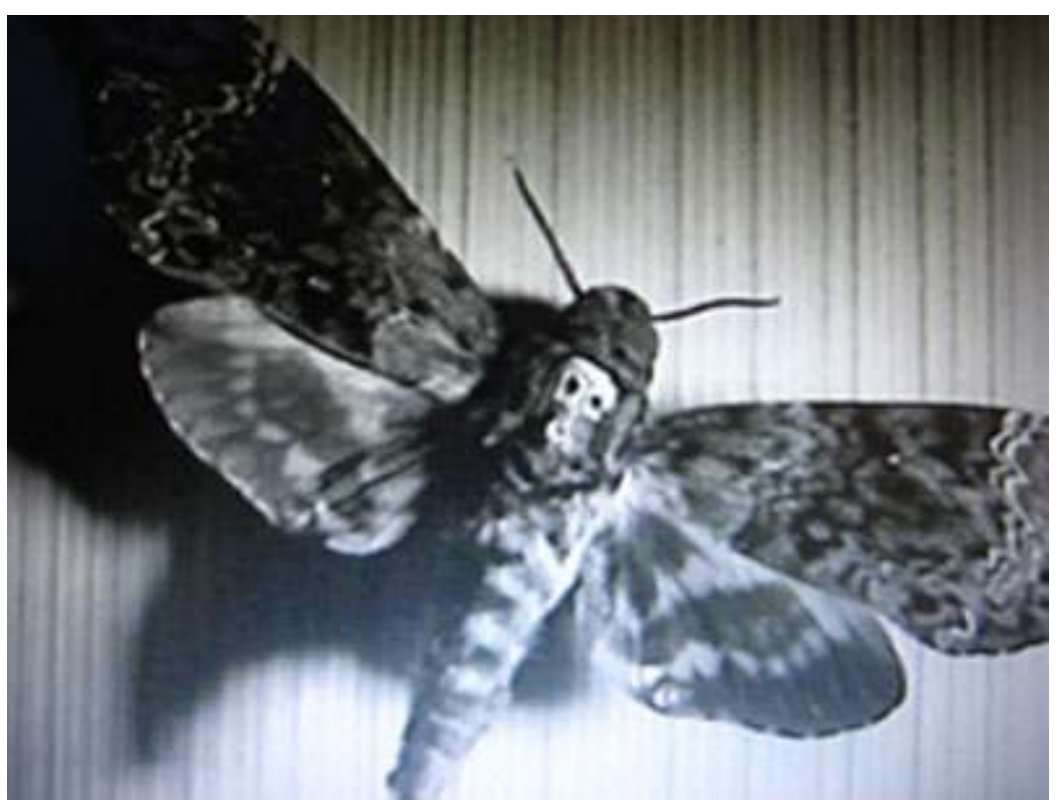

Luis Buñuel et Salvador Dali, Un chien andalou, 1929.

Ainsi, les techniques photographiques et cinématographiques s'accordent avec une vision de l'histoire dont elles vont alimenter métaphoriquement la description, chez Kracauer notamment qui, dans ces derniers écrits, « réali[se] en un éclair les multiples parallèles que l'on peut tracer entre l'histoire et les médias photographiques $»^{47}$.

Entre papillon et tête de mort, l'image photographique et/ou cinématographique est pareille au fameux Acherontia atropos rendu célèbre par Luis Buñuel et Salvador Dali (fig. 15).

Elle possède un caractère proprement épiphanique qui donne son modèle à l'image dialectique, « ce en quoi l'Autrefois rencontre le Maintenant dans un éclair pour former une constellation $\star^{48}$. Car, s'il est «un déclin de l'aura», W.Benjamin constate également que «la plus exacte technique peut donner à ses productions une valeur magique qu'aucune image peinte ne saurait plus avoir à nos yeux. Malgré toute la maitrise du photographe, malgré l'attitude composée de son modèle, le spectateur se sent forcé malgré lui de chercher dans une telle photo la petite étincelle de hasard, d'ici et maintenant, grâce à laquelle le réel a pour ainsi dire brûlé un trou dans l'image ; il cherche à trouver le lieu imperceptible où, dans la qualité singulière de cette minute depuis longtemps révolue, niche aujourd'hui encore l'avenir, d'une manière si éloquente que nous pouvons le découvrir rétrospectivement $»^{49}$. Cette apparition de l'instant passé dans l'image photographique et/ou cinématographique est une expérience de la mort qui est également une vision allégorique : en effet, c'est dans les images de la modernité que le crâne surgit comme le notait Kracauer en 1940 à Marseille. L'apparition des morts, c'est l'apparition de la tête de mort, ce que W.G. Sebald, commentant l'ouvrage de Hanns Zischler, Kafka geht ins Kino, décrit parfaitement : «Inondés de stimuli visuels comme nous le sommes aujourd'hui, il nous est difficile de comprendre la fascination que les images de cinéma pouvaient exercer, du temps de Kafka, sur ceux qui étaient prêts à s'abandonner complètement à l'illusionnisme d'un art encore primitif à bien des égards, que les arbitres du bon goût considéraient comme inférieur. Mais Zischler, peut-être parce qu'il a lui-même été 
devant la caméra, sait exactement ce qu'il en est de cet affect bizarrement mêlé de douleur identificatoire et d'aliénation, qui consiste dans le fait que dans le cas extrême, et néanmoins fréquent au cinéma, on se voit soi-même mourir. $»^{50}$

\section{Demonstratio ad oculos}

L'histoire naturelle chez $\mathrm{W}$. Benjamin convoque donc un réseau d'images dont la fonction est allégorique. Elles livrent une vision de l'histoire comme destin inéluctable, souffrance sans fin. Le genre baroque de la Vanité est alors comparable aux images photographiques et cinématographiques dont W. Benjamin fait également les dépositaires de la vision allégorique. Au cœur des guerres, les images de la modernité ont pour charge - comme déjà les Vanités - de restituer notre " peur de la mort $»^{51}$. Et pour cause : ce sont des parangons de l'image dialectique, et elles inventent un regard critique qui « dévoile tout, qui transperce tout [...] où le temps concret est aboli et où, comme parfois dans le rêve, les défunts, les vivants et ceux qui ne sont pas encore nés se retrouvent sur le même plan $\aleph^{52}$. Si l'historien de l'art ne peut désormais ignorer ce regard, il implique également sa discipline dans une théorie de la connaissance historique que $\mathrm{W}$. Benjamin définit comme histoire de la nature. Car c'est dans les images de la modernité, comme dans les Vanités, qu'apparaît «la physionomie allégorique de l'histoire-nature ${ }^{53}$ ».

Fig. 16

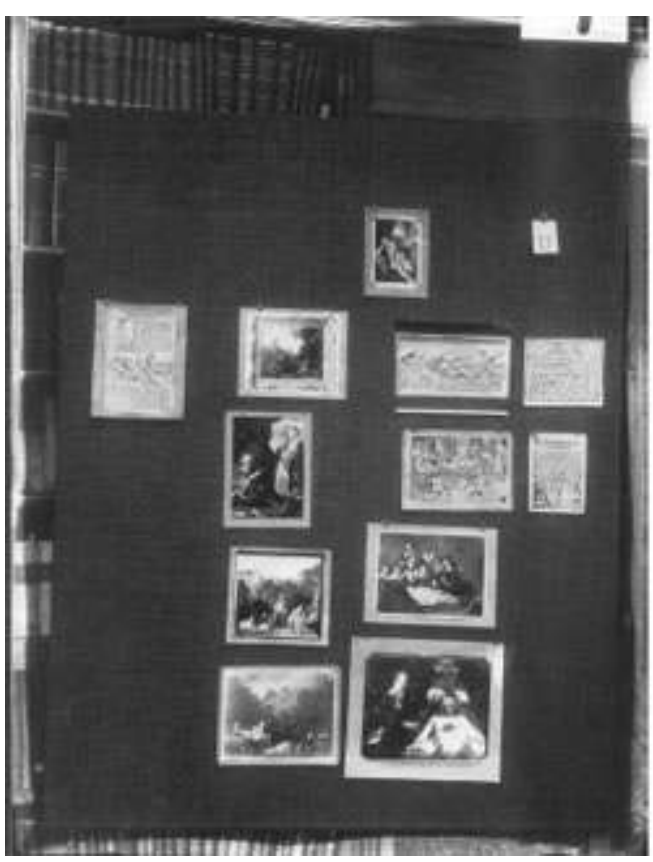

Aby Warburg, Der Bilderatlas Mnemosyne, planche 75.

Il est en réalité question d'une demonstratio ad oculos ${ }^{54}$ dont le tableau de Rembrandt est une parfaite illustration: de sa pince, il montre, exhibe l'intériorité découverte, un fragment d'atlas anatomique comme le remarque W.G. Sebald. Tel est le geste linguistique et iconographique qui s'impose dans le tableau de Rembrandt, et qui situe la métaphore de l'anatomie chez $\mathrm{W}$. Benjamin dans sa conception de la connaissance historique : ne démontrer que de montrer, et cela grâce à une méthodologie qui s'inspire 
des techniques de la modernité. «Méthode de ce travail : le montage littéraire. Je n'ai rien à dire. Seulement à montrer. Je ne vais rien dérober de précieux, ni m'approprier des formules spirituelles. Mais les guenilles, le rebut ${ }^{55}$ ». Propos d'anatomiste. Le regard nu comme une lame, il cherche la cause de cette folie destructrice qu'est l'histoire. Les rebuts, les guenilles, ces documents-déchets, ces entrailles de l'histoire, W. Benjamin les fouille semblable à un Démocrite éventrant des animaux, à l'ombre d'un platane, pour trouver l'organe qui produit la folie. Cette scène de la vie du philosophe grec, marquée par la rencontre de ce dernier avec Hippocrate, Aby Warburg la rend indissociable de la Leçon d'anatomie du Docteur Nicolaas Tulp de Rembrandt dans la planche 75 (fig. 16) de son Bilderatlas Mnemosyne ${ }^{56}$.

Ce dernier est un montage de plus de mille images reproduites sur une petite centaine de planches, demonstratio ad oculos. Avec la planche 75, l'atlas d'images devient atlas d'anatomie, constellation de scènes où se réunissent des hommes autour d'un mort dans des œuvres d'art appartenant aux $15^{\mathrm{e}}, 16^{\mathrm{e}}$ et $17^{\mathrm{e}}$ siècles. Des corps d'animaux, le corps du Christ, celui d'un Condottiere, d'Aris Kindt, d'un anonyme dans la Leçon du Dr John Deyman (1656) qui figure sous la toile 1632. Le dénominateur commun n'est donc pas seulement l'opération anatomique, c'est également un corps sacrifié ; dans trois des quatre versions de la scène de la rencontre entre Hippocrate et Démocrite, formant une colonne d'images à gauche de la planche de A. Warburg, c'est l'agneau ouvert les pattes ligotées. À côté de ce dernier, Démocrite est en train d'écrire un traité sur la folie; il procède littéralement à une anatomie de la mélancolie. On voit également dans cette planche 75 les apôtres Pierre et Paul qui apparaissent dans la seule reproduction échappant au motif du corps, placée comme en exergue, en haut à droite. Chacun représente respectivement l'apôtre des Juifs et l'apôtre des Gentils, manière de figurer une alliance entre christianisme et paganisme qui, chez Warburg, se manifeste grâce à la survivance de ce qu'il nomme des formules de pathos ${ }^{57}$, homologies formelles de l'extrême émotion dont le sémantisme s'inverse à travers les siècles. En premier lieu, donc, le point commun de ces scènes est un corps chu, lourd, mort : le corps du Christ de la mise au tombeau, celui d'un Condottiere, d'un Aris Kindt. Prophète, guerrier, larron se tiennent sur le théâtre d'un pathos qui n'a d'équivalent, dans sa puissance de démonstration, que le regard objectif et froid de l'anatomiste. À l'aune de ce montage, la dimension rituelle des scènes d'anatomie s'impose (lavement/purification, dissection/démembrement), les vivants s'affairant autour de cadavres avec frénésie ou calme, selon des modes dont la contradiction trouve pourtant dans le corps étendu un invariant inéluctable. Autour du mort évoluent femmes ou médecins, pleureuses ou anatomistes, Dionysos ou Apollon, les deux arcanes de la tragédie. Un peu plus à la gauche des spectateurs de Mnemosyne, c'est le fou qui ouvre des bestiaux mais aussi, tout à gauche de la planche, les oracles. En second lieu, donc, comme le montre une page extraite d'un ouvrage sur l'art de l'aruspice (Leberschau), le geste d'ouvrir le corps permet aussi de lire l'avenir dans les entrailles des animaux sacrifiés. Autrement dit, pour W. Benjamin procédant au découpage et au montage de fragments : lire le passé dans les entrailles de l'histoire comme une prophétie.

30 A. Warburg nous apprend ainsi que le regard anatomiste, dont use métaphoriquement W. Benjamin pour pointer la spécificité du regard moderne est inscrit dans la filiation d'un regard archaïque, le regard devin, qui déchiffre la nature autrement que les textes : en l'occurrence grâce à la disposition des organes. La spécificité de cette lecture est de prendre en compte la puissance de la physis et de relier l'homme à l'espace 
naturel. Si, chez W. Benjamin, il est question de l'histoire comme prophétie (« on dirait qu'il veut élucider les énigmes que les cabines de bateau ou les roulottes de bohémiens posent à l'enfant étonné $\left.{ }^{58} »\right)$, c'est en vertu de sa conception dialectique du rapport entre l'histoire et la nature. Anatomiser, ce n'est donc pas seulement ouvrir un cadavre, analyser oumétaphoriser la nouveauté d'un regard, c'est se saisir de la lisibilité du monde ${ }^{59}$ comme une histoire naturelle des images dont la signification circule du corps humain au corps astral, dialectise l'intimité et l'immensité, le viscéral et le céleste.

\section{NOTES}

1. Rainer Maria Rilke, Les carnets de Malte Laurids Brigge, trad. Claude David. Paris, Gallimard, 1996, p. 151.

2. W.G. Sebald, De la destruction comme élément de l'histoire naturelle, trad. Patrick Charbonneau. Arles, Actes Sud, 2005 (1re éd. all. 1999).

3. Id., Les Anneaux de Saturne (1995), trad. Bernard Kreiss, 1999. Paris, Gallimard, 2003, "Folio ", p. 27 (1 ${ }^{\text {re }}$ éd. all. 1995). Cf. Muriel Pic, W.G. Sebald. L'Image papillon, suivi de W.G. Sebald, L'Art de voler. Paris, Les Presses du Réel, 2009.

4. Ibid., p. 27.

5. Ibid., p. 25.

6. Walter Benjamin, L'Euvre d'art à l'époque de sa reproductibilité technique, in Euvres III. Paris, Gallimard, «Folio », p. 278, note.

7. Sur ce geste de Vésale, voir Georges Didi-Huberman, « Main dans la main du mort », préface à Raphaël Cuir, The

Renaissance on Anatomy. From Theology to Cartesianism, Londres, 2009 (à paraître)

8. Walter Benjamin, L'Euvre d'art à l'époque de sa reproductibilité technique, in Euvres III p. 301.

9. Walter Benjamin, Origine du drame baroque allemand, trad. Sybille Muller. Paris, Flammarion, «Champs », 2000, p. 178-179.

10. Cité par Miriam Hansen dans son introduction « With Skin and Hair » à Siegfried Kracauer, Theory of Film. The Redemption of Physical reality. Princeton, Princeton University Press, 1997. Citation reprise par Carlo Ginzburg, "Détails, gros plan, micro-analyse », in Siegfried Kracauer, penseur de l'histoire, dir. Philippe Despoix \& Peter Schöttler. Paris, Éd. de la Maison des sciences de l'homme/Sainte-Foy, Presses de l'Université Laval, 2006, p. 45-64.

11. Max Horkheimer \& Theodor W. Adorno, «La production industrielle de biens culturels : raison et mystification des masses», in La Dialectique de la raison, trad. Eliane Kaufholz. Paris, Gallimard, 2004 (1 ${ }^{\text {re }}$ éd. all. 1944), p. 129-176.

12. Walter Benjamin, "Théâtre et radio", Essais sur Brecht, trad. Philippe Ivernel, ed. Rolf Tiedemenn. Paris, La Fabrique, 2003, p. 125.

13. Id., Petite histoire de la photographie, in Cuvres II. Paris, Gallimard, « Folio », p. 295-321.

14. Id., L'Euvre d'art à l'époque de sa reproductibilité technique (1939), in Euvres III, op. cit., p. 300.

15. Louis-Ferdinand Céline, Nord, in CEuvres complètes II, éd. Henri Godard. Paris, Gallimard « La Pléiade ", 2003, p. 333 : « aussi ce qu'était assez curieux c'est que sur chaque trottoir, [...] tous les décombres [...] étaient amoncelées, impeccables, pas en tas n'importe comment, chaque maison avait ses débris devant sa porte, à la hauteur d'un, deux étages... et des débris numérotés !... que demain la guerre aille finir, subit..., il leur faudrait pas huit jours pour remettre tout en place... 
[...] là Berlin, huit jours, ils remettaient tout debout !... la maison bien morte, qu'un cratère, tous ses boyaux, tuyaux hors, la peau, le cœur, les os, mais tout son dedans n'empêche en ordre, bien agencé, sur le trottoir... comme l'animal aux abattoirs... ». Par ailleurs, c'est sur le registre de l'anatomie également que Pierre Mac Orlan a comparé le Voyage au bout de la nuit à Berlin Alexanderplatz : ce roman « ressemble à une opération chirurgicale [...] Alfred Döblin a posé sur sa table de travail un morceau de viande vivante, un morceau de chair berlinoise ", cité par Philippe Roussin, Misère de la littérature, terreur de l'histoire: Céline et la littérature contemporaine. Paris, Gallimard, 2005, p. 158.

16. Bertolt Brecht, "Sur le cinéma », Sur le cinéma, trad. Jean-Louis Lebrave et Jean-Pierre Lefebvre. Paris, L'Arche, 1970, pp. 199-200.

17. Walter Benjamin, Petite histoire de la photographie, in Euvres II, op. cit., p. 312.

18. W. H. F. Talbot, Le Crayon de la Nature, trad. Philippe Poncet. Paris, Les éditions de l'Amateur, 2003, p. 103..

19. Walter Benjamin, L'Euvre d'art à l'époque de sa reproductibilité technique, in CEuvres III, op. cit., p. 300.

20. Id., Petite histoire de la photographie, in CEuvres II, op. cit., p. 301. Le lecteur trouvera un énoncé semblable dans L'Euvre d'art à l'époque de sa reproductibilité technique, Euvres III, op. cit., p. 306.

21. Id., Petite histoire de la photographie, in CEuvres II, op. cit., p. 301.

22. Walter Benjamin, L'Cuvre d'art à l'époque de sa reproductibilité technique, in Cuvres III, op. cit., p. 304.

23. Sur ces questions, voir Rafael Mandressi, Le Regard de l'anatomiste. Paris, Le Seuil, 2004, p. 95-108.

24. Walter Benjamin, Origine du drame baroque allemand, op. cit., p. 195.

25. Id., L'Euvre d'art à l'époque de sa reproductibilité technique, in EEuvres III, op. cit., p. 300. Je souligne.

26. Jean Epstein, Photogénie de l'impondérable. Paris, Éditions Corymbe, 1935, p. 9-10.

27. Johann Wolfgang von Goethe, Lettre à Hetzler le jeune du 14 juillet 1770, Strasbourg, in «Correspondance », Appendice à La Métamorphose des plantes, trad. Henriette Bideau, éd. de PaulHenri Bideau. Paris, Triades, 1999, p. 321.

28. Walter Benjamin, «La Chasse aux papillons », in Sens Unique, trad. Jean Lacoste. Paris, 10/18, 2000 (1re éd. 1933), p. 24. Cf. Muriel Pic, «Image-papillon et ralenti. W.G. Sebald ou le regard capturé », Inframince (Arles), n², 2006, p. 90-104. Sur l'image comme papillon, voir aussi Georges Didi-Huberman, La Imagen mariposa, trad. J.J. Lahuerta. Barcelone, Ed. Muditito, 2007, et « Dessin, désir, métamorphose (esquissés sur les ailes d'un papillon)», in Le Plaisir au dessin. Carte blanche à Jean-Luc Nancy, s. dir. É. Pagliano \& S. Ramond. Lyon- Musée des Beaux-Arts de Lyon/ Paris, Hazan, p. 215-226.

29. W. Benjamin Sur le concept d'histoire, in Euvres III, op. cit., p. 432.

30. Id., Paris, capitale du XIX $x^{e}$ siècle : le livre des passages, trad. Jean Lacoste, d'après l'éd. originale de Rolf Tiedemann. Paris, Éd. du Cerf, 2006, p. 476.

31. Sur le papillon comme imago, cf.Georges Didi-Huberman, «Limage brûle», in Penser par les images: autour des travaux de Georges Didi-Huberman, s.dir. L. Zimmerman. Nantes, Éd. Cécile Defaut, 2006, p. 17-18. Muriel Pic, « Image-papillon et ralenti. W.G. Sebald ou le regard capturé », op. cit.

32. Virginia Woolf, La Mort de la phalène, trad. Hélène Bokanowski. Paris, Le Seuil, « Points », 2004

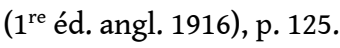

33. W. Benjamin, L'CEuvre d'art à l'époque de sa reproductibilité technique, in đEuvres III, op. cit., p. 290.

34. Id., Charles Baudelaire: un poète lyrique à l'apogée du capitalisme, trad. et préf. Jean Lacoste, d'après l'éd. originale établie par Rolf Tiedemann. Paris, Payot \& Rivages, 2002, p. 83. Karl Marx, Le Capital (1876), T. I, trad. Joseph Roy. Paris, Éditions sociales, 1978, p. 86. 
35. Jean Starobinski, «La leçon d'anatomie ", préface à Robert Burton, Anatomie de la mélancolie. Paris, Corti, 2000, p. XII. Sur la révolution du regard clinique, cf. Michel Foucault, Naissance de la clinique. Paris, PUF, 1963.

36. Walter Benjamin, Origine du drame baroque allemand, op. cit., p. 196

37. Ibid., p. 178-179.

38. Ibid., p. 191.

39. Theodor Adorno, "Die Idee der Naturgeschichte", in Philosophische Frühschriften, éd. Rolf Tiedemann. Frankfurt, M., Suhrkamp, 2003, p. 360. Cf. L'Actualité de la philosophie et autres essais, trad. s. dir. de Jacques-Olivier Bégot. Paris, Éditions ENS rue d'Ulm, 2008, pp. 31-55.

40. Heinrich Böll, Le Silence de l'ange, trad. Alain Huriot. Paris, Seuil, 1995 (1 ${ }^{\text {re }}$ éd. all. 1992), p. 91.

41. W. G. Sebald, Austerlitz, trad. Patrick Charbonneau. Paris, Gallimard, 2006 (1 ${ }^{\text {re }}$ éd. all. 2001), p. 183.

42. Id., Paris, capitale $d u$ XIX $X^{e}$ siècle, op. cit., p. 337. Voir également dans Origine du drame baroque allemand, op. cit, p. 235 : «Il y a dans la physis, dans la mnémè elle-même, un memento mori qui reste en éveil ».

43. Id., Origine du drame baroque allemand, op. cit., p. 202.

44. Ibid., p. 46.

45. Sigrid Weigel, Walter Benjamin: die Kreatur, das Heilige, die Bilder. Frankfurt am Main, Fischer Taschenbuch Verl., 2008, p. 306-307.

46. Walter Benjamin, Paris, capitale du XIXe siècle, op. cit., p. 489.

47. Siegfried Kracauer, L'Histoire des avant-dernières choses, trad. Claude Orsoni, éd. Nia Perivolaropoulou \& Philippe Despoix. Paris, Stock, 2006, p. 55.

48. Walter Benjamin, Paris, capitale du XIXe siècle, op. cit., p. 478.

49. Id., Petite histoire de la photographie, in Euvres II, op. cit., p. 300.

50. W.G. Sebald, Campo Santo, trad. Patrick Charbonneau \& Sybille Muller. Arles, Actes Sud, 2009, p. 191.

51. Siegfried Kracauer, «La photographie », in Le voyage et la danse: figures de villes et vues de films, trad. Sabine Cornille. Saint-Denis, Presses universitaires de Vincennes, p. 53.

52. W.G. Sebald, Campo Santo, op. cit., p. 192.

53. Walter Benjamin, Origine du drame baroque allemand, op. cit., p. 190.

54. Le terme vient du linguiste Bühler auquel Benjamin consacre un long moment de son essai sur « La sociologie du langage », Euvres III, op. cit., p. 30.

55. Walter Benjamin, Paris, capitale du XIXe siècle. Le Livre des passages, op. cit., p. 476.

56. Aby Warburg, Gesammelte Schriften II. 1, éd. Martin Warnke. Berlin, Akademie Verlag, 2008.

57. Giovanni Careri, Aby Warburg, «Rituel, Pathosformel et forme intermédiaire », in L'Homme. Image et anthropologie, $\mathrm{n}^{\circ} 165$, janvier-mars 2003, Paris, EHESS. http://lhomme.revues.org/ index198.html

58. Theodor W. Adorno, Sur Walter benjamin, trad. Sibylle Muller. Paris, Allia, 1999, p. 30.

59. Hans Blumenberg, La Lisibilité du monde, trad. Pierre Rusch et Denis Trierweiler. Paris, Cerf, 2007.

\section{INDEX}

Mots-clés : regard anatomiste, Sebald (Winfried Georg), Warburg (Aby) 


\section{AUTEUR}

\section{MURIEL PIC}

Docteur de l'EHESS, Muriel Pic enseigne la littérature française à l'université de Neuchâtel et a mené ses recherches sur le montage littéraire à l'université libre de Berlin. 